\title{
INVARIANTS OF IDEALS HAVING PRINCIPAL REDUCTIONS
}

\author{
Marco D'Anna, Anna Guerrieri and William Heinzer
}

\begin{abstract}
For a regular ideal having a principal reduction in a Noetherian ring we consider the structural numbers that arise from taking the Ratliff-Rush closure of the ideal and its powers. In particular, we analyze the interconnections among these numbers and the relation type and reduction number of the ideal. We prove that certain inequalites hold in general among these invariants, while for ideals contained in the conductor of the integral closure of the ring we obtain sharper results that do not hold in general. We provide applications to the one-dimensional local setting and present a sequence of examples in this context.
\end{abstract}

\section{Introduction.}

Given a regular ideal $I$ in a Noetherian ring $R$ it is possible to construct several filtrations and, in turn, various graded rings whose structures provide information about the geometrical properties of $I$. Two very well-known examples are the $I$-adic filtration and the filtration obtained by taking the Ratliff-Rush closure of the powers of $I$, the so called Ratliff-Rush filtration. A wealth of work has been produced, in the past years, on these filtrations, especially because of the role they play in the theory of the Hilbert function of $I$ when $R$ is a local ring with maximal ideal $\mathfrak{m}$ and $I$ is $\mathfrak{m}$-primary. These two filtrations coincide asymptotically but can differ greatly in the first steps. To be able to control these differences means to be able to control the properties of the associated graded ring of $I$. For example, the associated graded ring of $I$ contains a regular element if and only if the $I$-adic filtration and the Ratliff-Rush filtration coincide from the very first step.

In the present work we study the interplay between these two filtrations when $I$ is a regular ideal having a principal reduction; if $R$ is local this corresponds to the case in which the special fiber of the Rees ring of $I$, the fiber cone of $I$, is a one-dimensional graded ring. (For a characterization of analytic spread one ideals in local rings see [RR2].)

To carry out this work, we analyze the interconnections among some structural numbers that arise from taking the Ratliff-Rush closure of $I$ and of its powers and some classical invariants related to $I$ : the reduction number and the relation type. For example, we investigate the mutual relations among the following, where $\mathbb{N}$ 
denotes the nonnegative integers:

$$
\begin{aligned}
& r=r(I)=\min \left\{n \in \mathbb{N} \mid I^{n+1}=x I^{n} \text { for some } \mathrm{x} \in I\right\} \\
& k=k(I)=\min \left\{n \in \mathbb{N} \mid \widetilde{I}=\left(I^{n+1}: I^{n}\right)\right\} \\
& h=h(I)=\min \left\{n \in \mathbb{N} \mid \widetilde{I^{m}}=I^{m} \text { for all } m \geq n\right\} .
\end{aligned}
$$

The integer $r$ denotes the reduction number of $I$. In defining $k$, we are using the Ratliff-Rush closure $\widetilde{I}$ of $I$, which is by definition

$$
\widetilde{I}:=\bigcup_{n \in \mathbb{N}}\left(I^{n+1}:_{R} I^{n}\right)
$$

We refer to the paper of Ratliff and Rush [RR1] where this concept was first introduced for a description of other properties of this object. We call $\widetilde{I}$ a Ratliff-Rush ideal, and refer to the integer $k$ as the Ratliff-Rush number of I. In [RR1, Remark (2.3)] it is proved that $\widetilde{I^{m}}=I^{m}$ for all sufficiently large integers $m$, i.e., all sufficiently high powers of $I$ are Ratliff-Rush ideals. This motivates our definition of $h$. We call $h$ the asymptotic Ratliff-Rush number of $I$.

The starting point of our analysis is the observation that if $I$ has a principal reduction, then $h \leq r$ and $k \leq r-1$, see Proposition and Proposition . These are facts that can be found in the literature (e.g. [RV]) in the case of the maximal ideal of a one-dimensional local ring.

When $R$ is a reduced Noetherian ring with total ring of fractions $Q(R)$ and the integral closure $\bar{R}$ of $R$ in $Q(R)$ is a finitely generated $R$-module, we obtain sharper results when $I$ is contained in the conductor of $\bar{R}$ into $R$. In this case, for example, $k=r-1$ (Theorem ) and, if $I$ does not coincide with its Ratliff-Rush closure, $h=r$ (Proposition). Again, we prove that for all $n \geq 0$

$$
\widetilde{I^{n+1}} \cap I^{n}=I^{n} \widetilde{I}
$$

see Thorem . We remark that this gives the analogue for the Ratliff-Rush filtration, under the current hypotheses, to the equalities shown by Itoh, [I], and by Huneke, $[\mathrm{H}]$, for the filtration of the integral closures of the powers of $I$. Always in this context we prove, see Corollary, that the reduction number of the filtration $\left\{\widetilde{I^{n}}\right\}_{n \geq 0}$ is less than or equal to 1 .

If $J \subseteq I$ is a reduction of $I$, i.e., $J I^{s}=I^{s+1}$ for some positive integer $s$, clearly we have

$$
\left(I^{2}: J\right) \subseteq\left(I^{s+2}: J I^{s}\right)=\left(I^{s+2}: I^{s+1}\right) \subseteq \widetilde{I} .
$$


Furthermore, for each $n \in \mathbb{N}, J^{n}$ is a reduction of $I^{n}$ and $\left(I^{n+1}: J^{n}\right) \subseteq \widetilde{I}$. It follows that the ascending chain $I \subseteq\left(I^{2}: J\right) \subseteq\left(I^{3}: J^{2}\right) \subseteq \cdots \subseteq\left(I^{m+1}: J^{m}\right) \subseteq \cdots$ stabilizes with $\left(I^{m+1}: J^{m}\right)=\widetilde{I}$ for all sufficiently large $m$. We refer to

$$
k_{J}=\min \left\{n \in \mathbb{N} \mid \widetilde{I}=\left(I^{k_{J}+1}: J^{k_{J}}\right)\right\}
$$

as the Ratliff-Rush number of $I$ with respect to $J$. It is easy to see that $k_{J} \leq k$. We give in Corollary conditions under which $k_{J}=k$, and illustrate in Example that with $J$ a principal reduction of $I$, it sometimes occurs that $k_{J}<k$.

Another interesting structural number associated to the $I$-adic filtration is the relation type of $I$, here denoted as $N(I)$. Going back to ideas utilized by Huckaba in [Hu2] we describe the relation between the relation type and the reduction number of $I$. In particular we show that $N(I) \leq r+1$ (Proposition ) and that the equality holds when the ideal is 2-generated (Proposition ). In Discussion 2.13 we describe a family of 3-generated ideals contained in the conductor of a one-dimensional domain for which the difference between the reduction number and the relation type grows arbitrarly large.

The results we find can be translated to the case of a one-dimensional CohenMacaulay local ring. In this already well investigated context (see the analysis in [RV]) we find that, when $I$ is contained in the conductor, $k \leq \lambda(\widetilde{I} / I)$, see Proposition .

In section 2 of the present work we study the connections among the invariants of $I$ we just described, under the assumption that $I$ has a principal reduction. In section 3 we study the sharper behavior one obtains adding the condition $I$ contained in the conductor. In section 4 we provide applications to the one-dimensional setting as well as a set of examples in this context.

\section{Ideals having principal reductions.}

Suppose $I$ is a regular ideal of a Noetherian ring $R$ having the property that there exists $x \in I$ and an integer $n \geq 0$ such that $x I^{n}=I^{n+1}$. In this situation $x R$ is said to be a principal reduction of $I$. Notice that if $x R$ is a principal reduction of a regular ideal $I$, then $x$ is a regular element of $R$, i.e., $x$ is a nonzerodivisor of $R$. It follows from [Hu1, page 504] that if $(x)$ and $(y)$ are principal reductions of $I$, then $x I^{n}=I^{n+1}$ if and only if $y I^{n}=I^{n+1}$. Thus the reduction number $r(I)$ is independent of the principal reduction. 
If $R$ is local with maximal ideal $\mathfrak{m}$, and $I$ is an ideal of $R$ having a principal reduction, then the fiber cone $F(I)=\bigoplus_{n \geq 0} I^{n} / \mathfrak{m} I^{n}=\bigoplus_{n \geq 0} F_{n}$ is a one-dimensional graded ring over the field $K:=R / \mathfrak{m}$. An element $x \in I$ is a principal reduction of $I$ if and only if $F(I)$ is integral over its $K$-subalgebra generated by the image of $x$ in $F_{1}=I / \mathfrak{m} I$. In this local setting, if $I$ has a principal reduction, then the principal reductions of $I$ are precisely the minimal reductions. In general, a reduction $J$ of $I$ is a minimal reduction if and only if $J R_{\mathfrak{m}}$ is a minimal reduction of $I R_{\mathfrak{m}}$ for each maximal ideal $\mathfrak{m}$ of $R$. Thus if $I$ is a regular ideal having a principal reduction in a Noetherian ring $R$ and if $J$ is a minimal reduction of $I$, then $J$ is locally principal and hence invertible. Also $J I^{n}=I^{n+1}$ if and only if equality holds locally at each maximal ideal. Therefore the reduction number $r(I)$ is independent of the minimal reduction. However a regular ideal having a principal reduction in a Noetherian ring may also have minimal reductions which are not principal. For example, let $t$ be an indeterminate over a field $K$ and let $R=K\left[t^{2}, t^{3}\right]$. Then $\left(t^{2}\right)$ is a principal reduction of $I=\left(t^{2}, t^{3}\right)$ and $J=\left(t^{2}+t^{3}, t^{4}\right)$ is an invertible minimal reduction of $I$ that is not principal.

Proposition 2.1: $\quad$ Suppose $R$ is a Noetherian ring and $I$ is a regular ideal of $R$ having a principal reduction. Then $k \leq \max \{0, r-1\}$, where $k$ is the Ratliff-Rush number of $I$ and $r$ is the reduction number of $I$.

Proof: If $r=0$, then $I$ is principal and $k=0$. Assume that $I^{r+1}=x I^{r}$ with $r \geq 1$. We need to show that $\widetilde{I}=\left(I^{r}: I^{r-1}\right)$. It is enough to show that $\left(I^{r}: I^{r-1}\right)=$ $\left(I^{r+s}: I^{r+s-1}\right)$ for each integer $s \geq 1$. Since $I^{r+s}=x^{s} I^{r}$ and $I^{r+s-1}=x^{s-1} I^{r}$, it suffices to show $\left(I^{r}: I^{r-1}\right)=\left(I^{r+1}: I^{r}\right)$.

The inclusion $\left(I^{r}: I^{r-1}\right) \subseteq\left(I^{r+1}: I^{r}\right)$ is always true. To prove the reverse inclusion, let $y \in\left(I^{r+1}: I^{r}\right)$; since $x I^{r-1} \subseteq I^{r}$, then $y x I^{r-1} \subseteq y I^{r} \subseteq I^{r+1}=x I^{r}$. Since $x$ is a regular element, it follows that $y I^{r-1} \subseteq I^{r}$, or equivalently, $y \in\left(I^{r}\right.$ : $\left.I^{r-1}\right)$.

Proposition 2.2: $\quad$ Suppose $R$ is a Noetherian ring and $I$ is a regular ideal of $R$ having a principal reduction. Then $h \leq r$, where $h$ is the asymptotic Ratliff-Rush number of $I$ and $r$ is the reduction number of $I$.

Proof: By assumption there exists $x \in I$ such that $I^{r+1}=x I^{r}$. Let $n \in \mathbb{N}, n \geq r$. It is well known, see [RR, Proposition 2.6], that $\widetilde{I^{n}}=\left(I^{n+i}: I^{i}\right)$ for all sufficient 
large $i \in \mathbb{N}$. Since $n \geq r$ and $I^{r+1}=x I^{r}$, it follows that $I^{n+i}=x^{i} I^{n}$ for $i \geq 1$. Since $x$ and all its powers are regular elements, we obtain that $\left(I^{n+i}: x^{i}\right)=I^{n}$ for all integers $i \geq 1$. The conclusion now follows because $\left(I^{n+i}: I^{i}\right) \subseteq\left(I^{n+i}: x^{i}\right)$.

If one wants to consider a higher dimensional version of the previous results, one needs to deal with the following example due to Raghavan (Example 1.2 in [HJLS]): Let $K$ be a field and $R$ be the complete local 2-dimensional domain $K \llbracket x, y^{2}, y^{7}, x^{2} y^{5}, x^{3} y \rrbracket$. The ideal $I=\left(x, y^{2}\right) R$ is a parameter ideal and therefore has reduction number $r=0$. However, $I$ is not Ratliff-Rush closed. Note in fact that $x^{2} y^{5}$ belongs to $\left(I^{2}: I\right)$ but not to $I$.

Question 2.3: $\quad$ In a Noetherian local ring, do the results in Proposition 2.1 and Proposition 2.2 hold for ideals I that have minimal reductions generated by regular sequences?

Remark 2.4: We call a regular ideal $I$ in a Noetherian $\operatorname{ring} R$ stable if there exists $x \in I$ with $x I=I^{2}$. For a regular ideal in a one-dimensional local ring, this definition is equivalent to that of Lipman [L, (1.3) and (1.11)]. Among regular ideals having principal reductions, the stable ideals are the ideals having reduction number at most one. It is well-known that if $I$ is stable, then $I$ is a Ratliff-Rush ideal. Thus $r(I)=1$ implies $k(I)=0$, i.e., $\widetilde{I}=(I: R)=I$. This is true quite generally. In fact, see [VV], if $I$ has reduction number $r \leq 1$ then the depth of the associated graded ring $G(I)=R[I t] / I R[I t]$ of $I$ is positive, hence $I$ and all its powers are Ratliff-Rush ideals, cf. [HLS, (1.2)]. Thus if $I$ is stable, then $h(I)=0$. It is also well known, however, that there exist Ratliff-Rush ideals $I$ that are not stable. We illustrate this fact in our section of examples (Example).

Discussion 2.5: $\quad$ Suppose $I$ is a regular ideal of $R$ having a principal reduction $x R$. Since $I^{n}$ is a finitely generated faithful $R$-module, if $z \in Q(R)$ is such that $z I^{n} \subseteq I^{n}$, then $z$ is in the integral closure $\bar{R}$ of $R$. In this context, the blowing-up ring of $I$ may be defined as

$$
R^{I}:=\bigcup_{n \in \mathbb{N}}\left(I^{n}:_{Q(R)} I^{n}\right)=\bigcup_{n \in \mathbb{N}}\left(I^{n}: \bar{R} I^{n}\right) .
$$

Note that this is an increasing union. If $I^{r+1}=x I^{r}$, then for $n>r$ we have

$$
I\left(I^{n}:_{Q(R)} I^{n}\right)=I\left(x I^{n-1}:_{Q(R)} I^{n}\right)=x\left(I^{n}:_{Q(R)} I^{n}\right) .
$$


Therefore $I R^{I}=x R^{I}$. It is clear that $\left(I^{n}:_{Q(R)} I^{n}\right) \subseteq\left(I^{n} / x^{n}\right)$ for each positive integer $n$. We also have $\left(I^{r} / x^{r}\right) \subseteq \cup_{n=1}^{\infty}\left(I^{n}:_{Q(R)} I^{n}\right)$, for if $y=z / x^{r}$ with $z \in I^{r}$, then $\left(z / x^{r}\right) I^{2 r}=\left(z / x^{r}\right) x^{r} I^{r}=z I^{r} \subseteq I^{2 r}$. Hence $y \in\left(I^{2 r}:_{Q(R)} I^{2 r}\right)$. Therefore the following equalities hold:

$$
R^{I}=\bigcup_{n=1}^{\infty} \frac{I^{n}}{x^{n}}=\frac{I^{r}}{x^{r}}
$$

where the second equality is an equality of fractional ideals; cf. [L] and [HLS]. It follows that, if $\mathrm{I}$ is a regular ideal with a principal reduction $x R$, then, for any $s \geq r$

$$
\widetilde{I}=\left(I^{s+1}: I^{s}\right)=\left(x I^{s}:_{Q(R)} I^{s}\right) \cap R=x R^{I} \cap R=I^{s} x^{-s+1} \cap R
$$

where the first equality follows by the proof of Proposition 2.1.

Remark 2.6: If $x R$ is a principal reduction of $I$, then $x^{m} R$ is a principal reduction of $I^{m}$ for each $m \in \mathbb{N}$; moreover, the reduction number of $I^{m}$ is less than or equal to the reduction number of $I$. Indeed, if $I^{r+1}=x I^{r}$, then $\left(I^{m}\right)^{r+1}=x^{m}\left(I^{m}\right)^{r}$. Also, $I^{2 r}=x^{r} I^{r}$ and for each $m \geq r$ we have $I^{2 m}=x^{m} I^{m}$. Hence if $I$ is a regular ideal having a principal reduction and if $I$ has reduction number $r$, then $I^{m}$ is stable for every integer $m \geq r$, see [SV, Section 2] and [ES, Corollary 1, page 446]. This gives another proof for Proposition 2.2. Eakin and Sathaye [ES, page 446] define an ideal $I$ in a semilocal ring to be prestable if some power of $I$ is stable. In a general ring $R$, they define an ideal $I$ to be prestable if for each prime ideal $P$ of $R, I R_{P}$ is prestable in $R_{P}$. It is shown in [ES, Corollary 1, p. 446] that if $I$ is a prestable ideal of a local ring and if $I^{n}$ has $n$-generators, then $I^{n-1}$ is stable. The smallest positive integer $s$ such that $I^{s}$ is stable is related to the asymptotic Ratliff-Rush number $h(I)$ in that $h(I) \leq s$. The distinction here is that all powers of $I$ being Ratliff-Rush does not imply in general that $I$ is stable, so sometimes $h(I)<s$.

For a regular ideal $I$ of a one-dimensional Cohen-Macaulay local ring $(R, \mathfrak{m})$, Sally and Vasconcelos [SV, Section 2] define $n(I)$ to be the least positive integer $n$ such that $I^{n}$ is stable. They define the index of stability $s(R)$ of $R$ to be the sup of $n(I)$ as $I$ varies over the regular ideals of $R$ and prove that $s(R) \leq \max \{1, e-1\}$, where $e$ is the multiplicity of $R$. These results of [SV] and [ES] imply that $r=e-1$ is a global bound for the reduction number of regular ideals $I$ of a one-dimensional local Cohen-Macaulay ring $(R, \mathfrak{m})$ having multiplicity $e$. (This can be deduced too from the Hilbert function in the one-dimensional case). In view of Proposition 2.1 
and 2.2 the number $e-1$ also gives a global bound on the Ratliff-Rush number and asymptotic Ratliff-Rush number of the regular ideals of a one-dimensional local Cohen-Macaulay ring $R$ of multiplicity $e$.

In relation to these results, Huckaba in [Hu1, Question 2.6] raises the following question: Suppose $(R, \mathfrak{m})$ is a quasi-unmixed analytically unramified local ring having infinite residue field and multiplicity e. (Assume if needed that $R$ contains a field of characteristic zero.) Is it true that $r(I) \leq e-1$ for every regular ideal $I$ of $R$ of analytic spread one? It seems to be unknown whether there even exists a global bound on $r(I)$ for the ideals $I$ of $R$ having a principal reduction. Work of Vasconcelos in $[\mathrm{V}]$ proves the existence of such a global bound if there exists a bound on the arithmetic degree of the fiber cone of $I$ as $I$ varies over the regular ideals of $R$ that have a principal reduction.

In the next result we characterize the condition $h=r$.

Proposition 2.7: $\quad$ Suppose $I$ is a regular ideal having a principal reduction $x R$ with reduction number $r$ in a Noetherian ring $R$. Then

(1) $\left(I^{r}: x\right)=\widetilde{I^{r-1}}$,

(2) $h=r$ if and only if $x I^{r-1} \subsetneq I^{r} \cap x R$.

Proof: To show $\widetilde{I^{r-1}}=\left(I^{r}: x\right)$, it suffices to observe that $\left(I^{r+n}: x^{n+1}\right)=\left(I^{r}: x\right)$ for all $n \geq 0$. If $a x^{n+1} \in I^{r+n}=x^{n} I^{r}$, then, since $x$ is a regular element, we have $a x \in I^{r}$.

By Proposition 2.2, $h \leq r$. Hence $h=r$ if and only if $I^{r-1} \subsetneq \widetilde{I^{r-1}}=\left(I^{r}: x\right)$. The result now follows since $I^{r-1} \subsetneq\left(I^{r}: x\right)$ if and only if $x I^{r-1} \subsetneq I^{r} \cap x R$.

We now analyze the relation type of ideals having principal reductions. The Rees algebra of $I$ is the graded subalgebra $R[I t]=\bigoplus_{i \geq 0} I^{i} t^{i}$ of the polynomial ring $R[t]$. A presentation of the Rees algebra of $I$ is obtained as follows: if $I=\left(x_{1}, \ldots, x_{n}\right) R$, let $R\left[T_{1}, \ldots, T_{n}\right]$ be a graded polynomial ring over $R$, and consider the graded $R$ algebra homomorphism $\phi: R\left[T_{1}, \ldots, T_{n}\right] \longrightarrow R[I t]$ defined by $\phi\left(T_{i}\right)=x_{i} t$. Let $Q=\operatorname{Ker} \phi$. Then

$$
R\left[T_{1}, \ldots, T_{n}\right] / Q \cong R[I t]
$$

and $Q$ is the homogeneous ideal generated by all forms in $R\left[T_{1}, \ldots, T_{n}\right]$ that vanish when evaluated at the generators $\left(x_{1}, \ldots, x_{n}\right)$ of $I$. The relation type $N(I)$ of $I$ 
is the least bound on the degrees of the polynomials required to generate $Q$. It is independent of the choice of a generating set for $I$.

The following proof is similar to that given in [W, page 54] which in turns relies on ideas given in [Hu2].

Proposition 2.8: $\quad$ Suppose $I$ is a regular ideal having a principal reduction in a Noetherian ring $R$. Then $I$ has relation type $N(I) \leq r+1$, where $r$ is the reduction number of $I$.

Proof: Let $x \in I$ be such that $I^{r+1}=x I^{r}$. Extend $x=x_{1}$ to a system $\left(x_{1}, \ldots, x_{n}\right)$ of generators of $I$. Consider the presentation $R[I t] \cong R\left[T_{1}, \ldots, T_{n}\right] / Q$. For $m \in \mathbb{N}$, let $Q_{m} \subseteq Q$ be the ideal generated by all homogeneous forms $F \in R\left[T_{1}, \ldots, T_{n}\right]$ such that $F\left(x_{1}, \ldots, x_{n}\right)=0$ and $\operatorname{deg} F \leq m$. It suffices to prove for $i$ a positive integer and $F \in Q$ a homogeneous polynomial with $\operatorname{deg} F=r+1+i$, then $F \in Q_{r+i}$.

If $F \in\left(T_{1}\right) R\left[T_{1}, \ldots, T_{n}\right]$, then $F=T_{1} G$ where $G$ is a form of degree $r+i$. We have

$$
0=F\left(x_{1}, \ldots, x_{n}\right)=x_{1} G\left(x_{1}, \ldots, x_{n}\right) .
$$

Since $x=x_{1}$ is a regular element of $R, G\left(x_{1}, \ldots, x_{n}\right)=0$. Hence $G \in Q_{r+i}$, so $F=T_{1} G \in Q_{r+i}$ in this case.

In the general case write $F=T_{1} G_{1}+\cdots+T_{n} G_{n}$ where each $G_{j}$ is a homogeneous polynomial of degree $r+i$. For $j \geq 2$, let $g_{j}=G_{j}\left(x_{1}, \ldots, x_{n}\right)$. Since $i$ is positive $g_{j} \in I^{r+i}=x_{1} I^{r+i-1}$. Therefore $g_{j}=x_{1} h_{j}$ with $h_{j} \in I^{r+i-1}$. Let $H_{j} \in R\left[T_{1}, \ldots, T_{n}\right]$ be a form of degree $r+i-1$ such that $H_{j}\left(x_{1}, \ldots, x_{n}\right)=h_{j}$. It follows that $G_{j}-T_{1} H_{j}$ is a form of degree $r+i$ that is in $Q$ and hence in $Q_{r+i}$. Moreover

$$
F-\sum_{j=2}^{n} T_{j}\left(G_{j}-T_{1} H_{j}\right)=T_{1}\left(G_{1}+T_{2} H_{2}+\ldots+T_{n} H_{n}\right)
$$

is a homogeneous form of degree $r+1+i$ that is in both $Q$ and $\left(T_{1}\right) R\left[T_{1}, \ldots, T_{n}\right]$. By the previous case $F-\sum_{j=2}^{n} T_{j}\left(G_{j}-T_{1} H_{j}\right) \in Q_{r+i}$. Therefore $F \in Q_{r+i}$.

As in Theorem 2.4 of [Hu2] one has:

Proposition 2.9: $\quad$ Suppose $R$ is a Noetherian ring and $I=(x, y) R$ is a 2generated non-principal regular ideal having $x R$ as a principal reduction. Then $I$ has relation type $N(I)=r+1$, where $r$ is the reduction number of $I$. 
Proof: By Proposition 2.8, $N(I) \leq r+1$.

Consider the presentation $R[I t] \cong R\left[T_{1}, T_{2}\right] / Q$, where $\phi\left(T_{1}\right)=x t$ and $\phi\left(T_{2}\right)=$ $y t$. For $m \in \mathbb{N}$, let $Q_{m} \subseteq Q$ be the ideal generated by all homogeneous forms $F \in R\left[T_{1}, T_{2}\right]$ such that $F(x, y)=0$ and $\operatorname{deg} F \leq m$.

For $s \in \mathbb{N}, I^{s+1}=x I^{s}$ if and only if $y^{s+1} \in x I^{s}$ if and only if there exists a homogeneous polynomial $F \in R\left[T_{1}, T_{2}\right]$ such that $\operatorname{deg} F=s+1, F$ is monic as a polynomial in $T_{2}$ and $F(x, y)=0$, i.e., $F \in Q_{s+1}$. Since $r$ is the smallest integer such that $I^{r+1}=x I^{r}$, there exists such a polynomial $F$ for $s=r$, but not for $s<r$. Therefore $Q_{r}$ contains no forms that are monic in $T_{2}$, while $Q_{r+1}$ does contain such a form. It follows that $N(I)=r+1$.

The previous result is actually a special case of Observation 4.9 in [W]. We gave the proof for its simplicity and because no particular assumption on the ring is needed.

If $I$ is stable, we show next that the equality of (2.9) holds with no restriction on the number of generators of $I$.

Corollary 2.10: $\quad$ Suppose $I$ is a stable non-principal regular ideal having a principal reduction in a Noetherian ring $R$. Then $I$ has relation type $N(I)=2$.

Proof: By Proposition 2.8, $N(I) \leq 2$. By assumption, there exists $x \in I$ such that $x I=I^{2}$. Let $x=x_{1}, x_{2}, \ldots, x_{n}$ be generators of $I$ and let $R\left[T_{1}, \ldots, T_{n}\right] / Q=$ $R\left[x_{1} t, \ldots, x_{n} t\right]$ be a presentation of the Rees algebra of $I$, where $T_{i} \rightarrow x_{i} t$ for $i=1, \ldots n$.

Suppose $N(I)=1$. The equality $I^{2}=x_{1} I$ implies $x_{n}^{2}=x_{1} g$, where $g \in$ $I=\left(x_{1}, \ldots, x_{n}\right) R$. Thus there exists a degree 2 form $T_{n}^{2}-T_{1} G$ which vanishes at $x_{1}, \ldots, x_{n}$. Our assumption that $N(I)=1$ implies there are linear forms $H_{1}, \ldots, H_{s} \in Q$ such that $T_{n}^{2}-T_{1} G=F_{1} H_{1}+\cdots+F_{s} H_{s}$, where $F_{1}, \ldots, F_{s}$ are linear forms in $R\left[T_{1}, \ldots, T_{n}\right]$. Comparing the coefficients of the monomial $T_{n}^{2}$, we deduce that the coefficients of $T_{n}$ in the $H_{i}$ generate the unit ideal of $R$. Hence there exist $a_{i} \in R$ such that $a_{1} H_{1}+\cdots+a_{s} H_{s}$ is monic in $T_{n}$. Since this form vanishes at $x_{1}, \ldots, x_{n}$, it follows that $x_{n} \in\left(x_{1}, \ldots, x_{n-1}\right) R$. Therefore $I=\left(x_{1}, \ldots, x_{n-1}\right) R$. If $n-1>1$, a repetition of the argument yields $x_{n-1} \in\left(x_{1}, \ldots, x_{n-2}\right) R$. Therefore a simple inductive proof gives $I=x_{1} R$. This contradicts our hypothesis that $I$ is not principal. We conclude that $N(I)=2$. 
Discussion 2.11: Suppose $I=(x, y, z) R$ is an ideal in a Noetherian domain $R$ such that $x R$ is a principal reduction of $I$. If $y^{2} / x^{2}$ and $z^{2} / x^{2}$ are units of $R$, but $y z \notin x I$, then $I$ has reduction number $r(I)=2$ and relation type $N(I)=2<$ $r(I)+1$. Therefore it is not possible to extend Proposition 2.9 to a situation where $I$ is 3 -generated. We have $x I=\left(x^{2}, x y, x z\right) \subsetneq I^{2}$ since $y z \notin x I$. However, the fact that the elements $y^{2}$ and $z^{2}$ are in $x^{2} R \subseteq x I$ implies that $I^{3} \subseteq x I^{2}$, so $r(I)=2$.

To see that $N(I)=2$, consider the presentation $R\left[T_{1}, T_{2}, T_{3}\right] / Q=R[x t, y t, z t]$ of the Rees algebra of $I$, where $T_{1} \rightarrow x t, T_{2} \rightarrow y t$, and $T_{3} \rightarrow z t$. Since $r(I)=2$, $N(I) \leq 3$ by Proposition 2.8. To show $N(I) \leq 2$, we show that each form $F \in Q$ with $\operatorname{deg} F=3$ is in $Q_{2}$. Since $y^{2} / x^{2}:=a$ and $z^{2} / x^{2}:=b$ are units of $R, T_{2}^{2}-a T_{1}^{2}$ and $T_{3}^{2}-b T_{1}^{2}$ are in $Q_{2}$. Using these relations we can modify $F$ to another form $G$ of degree 3 that is in $Q$ and is a multiple of $T_{1}$. Thus $G=T_{1} H$, where $H \in R\left[T_{1}, T_{2}, T_{3}\right]$ is a form of degree 2. Since $G \in Q$, we have $0=G(x, y, z)=x H(x, y, z)$. Since $x$ is a regular element in $R$, it follows that $H(x, y, z)=0$. Therefore $H \in Q_{2}$, and so also $G$ and $F$ are in $Q_{2}$. We conclude that $Q_{2}=Q$ and $N(I)=2$.

Example 2.12: An example of a one-dimensional Noetherian domain $R$ having an ideal $I=(x, y, z) R$ with the properties of (2.11) may be constructed as follows: Let $F$ be a field having an algebraic extension $K=F(\alpha, \beta)$ such that $[K: F]=4$ and such that $\alpha^{2}=a \in F$ and $\beta^{2}=b \in F$. Let $x$ be an indeterminate over $K$ and let $R=F+x K[x]$. Thus $R$ is the set of polynomials in $K[x]$ that have their constant term in $F$. Let $y=\alpha x, z=\beta x$ and $I=(x, y, z) R$. (For example, $F$ could be the field $\mathbb{Q}$ of rational numbers, $\alpha$ could be the square root of 2 and $\beta$ the square root of 3.) Since $1, \alpha, \beta, \alpha \beta$ is a vector space basis for $K$ over $F, y z=\alpha \beta x^{2}$ is not in $x I$. Notice that $K[x]$ is the integral closure of $R$ and $I$ is contained in $x K[x]$ which is the conductor of the integral closure of $R$ into $R$. Localization of $R$ at the maximal ideal $x K[x]$ gives a one-dimensional local Noetherian domain with the same properties.

Discussion 2.13: The examples displayed so far describe ideals for which the relation type is bounded below by the reduction number. A modification of the construction of (2.12) gives for each integer $n \geq 3$ an ideal $I=(x, y, z) R$ in a one-dimensional Noetherian domain $R$ having a principal reduction $x R$ such that $I$ has reduction number $r=2 n-2$ and relation type $N(I)=n$. Thus there exist 3 generated ideals having principal reductions and having the difference between the 
reduction number and the relation type arbitrarily large; in fact $r-N(I)=n-2$.

To construct such examples, fix $n \geq 3$ and let $F$ be a field having an algebraic extension $K=F(\alpha, \beta)$ such that $[K: F]=n^{2}$ and such that $\alpha^{n}=a \in F$ and $\beta^{n}=b \in F$. Let $x$ be an indeterminate over $K$ and let, as before, $R=F+x K[x]$. Let $y=\alpha x, z=\beta x$ and $I=(x, y, z) R$. The set $\left\{\alpha^{i} \beta^{j}\right\}$, where $0 \leq i, j \leq n-1$, is a vector space basis for $K$ over $F$ and a module basis over $R$ for the integral closure $K[x]$ of $R$. It follows that $y^{n-1} z^{n-1}=\alpha^{n-1} \beta^{n-1} x^{2 n-2}$ does not belong to $x I^{2 n-3}=x(x, \alpha x, \beta x)^{2 n-3}$. Thus $x I^{2 n-3}$ is properly contained in $I^{2 n-2}$. Moreover, the elements $y^{n}, z^{n}$ belong to $x^{n} R$ and we get $I^{2 n-1}=(x, y, z)^{2 n-1}=x I^{2 n-2}$. Therefore $r(I)=2 n-2$.

To see that $N(I)=n$, consider the presentation $R\left[T_{1}, T_{2}, T_{3}\right] / Q=R[x t, y t, z t]$ of $R[I t]$. Since $r(I)=2 n-2, N(I) \leq 2 n-1$ by Proposition 2.8. To show $N(I) \leq n$, we show that each form $G \in Q$ of degree $m$ with $n \leq m \leq 2 n-1$, is in $Q_{n}$. Since the relations $x T_{2}-x \alpha T_{1}, x T_{3}-x \beta T_{1}$ are in $Q_{1}$, we may assume that

$$
G\left(T_{1}, T_{2}, T_{3}\right)=\sum_{i+j+k=m} r_{i j k} T_{1}^{i} T_{2}^{j} T_{3}^{k} \in R\left[T_{1}, T_{2}, T_{3}\right]
$$

has the property that $r_{i j k} \in F$ if $j+k>0$. Using the relations $T_{2}^{n}-a T_{1}^{n}, T_{3}^{n}-b T_{1}^{n} \in$ $Q_{n}$, we may assume $j<n$ and $k<n$ for each nonzero $r_{i j k}$. Then

$$
G(x, \alpha x, \beta x)=\sum_{i+j+k=m} r_{i j k} x^{i}(\alpha x)^{j}(\beta x)^{k}=0
$$

implies that

$$
r_{m 00}(0)+\sum_{j+k>0, j<n, k<n} r_{i j k} \alpha^{j} \beta^{k}=0 .
$$

The linear independence over $F$ of $\left\{\alpha^{j} \beta^{k}\right\}, 0 \leq j, k \leq n-1$ implies that $r_{m 00}$ and each of the $r_{i j k}$ is zero. It follows that $G\left(T_{1}, T_{2}, T_{3}\right)=0$. Therefore $N(I) \leq n$. On the other hand, the relations $T_{2}^{n}-a T_{1}^{n}$ and $T_{3}^{n}-b T_{1}^{n}$ are monic in $T_{2}$ and $T_{3}$ and are easily seen to be in $Q_{n}$ and not in $Q_{n-1}$. In conclusion, we obtain $N(I)=n$.

Observe that the ideals constructed in (2.13) are contained in the conductor of $R$ in its integral closure. We show, in the next section, that other invariants of ideals contained in the conductor have a more predictable behaviour. The relation type however seems to remain unaffected with respect to containment in the conductor. 


\section{Reduced Noetherian rings with finite integral closure.}

Notation 3.1: $\quad$ Suppose now that $R$ is a reduced Noetherian ring having total ring of fractions $Q(R)$. Assume that the integral closure $\bar{R}$ of $R$ in $Q(R)$ is a finitely generated $R$-module. Let $\mathfrak{C}=\left(R:_{Q(R)} \bar{R}\right)$ denote the conductor of $\bar{R}$ into $R$. Since $\bar{R}$ is a finitely generated $R$-module, $\mathfrak{C}$ is a regular ideal of both $R$ and $\bar{R}$.

In this context, if $I \subseteq \mathfrak{C}$ is a regular ideal that is not principal but has a principal reduction, we prove in Theorem 3.2 that the Ratliff-Rush number $k$ of $I$ is precisely $r-1$, where $r$ is the reduction number of $I$. What allows us to prove a sharp equality result in this case (as contrasted with only an inequality in Proposition 2.1) is that, in this case,

$$
\widetilde{I}=I^{s} x^{-s+1} \cap R=I^{s} x^{-s+1}
$$

(for $s$ large enough); the last equality holds since $I^{s} x^{-s+1}=I R^{I} \subseteq R^{I} \subseteq \bar{R}$ and $I \subseteq \mathfrak{C}$ which is an ideal of $\bar{R}$; therefore $I R^{I} \subseteq \mathfrak{C} \subseteq R$.

Theorem 3.2: $\quad$ Suppose $R$ is a reduced Noetherian ring as in (3.1) and $I$ is a regular ideal of $R$ having a principal reduction. If $I \subseteq \mathfrak{C}$ and if $I$ is not principal, then the reduction number $r$ of $I$ is $k+1$, where $k$ is the Ratliff-Rush number of $I$.

Proof: By hypotheses $I^{r+1}=x I^{r}$ and $I^{r} \supsetneq x I^{r-1}$. Since $I$ is a regular ideal contained in $\mathfrak{C}$, it follows that

$$
I \subsetneq I^{2} x^{-1} \subsetneq \cdots \subsetneq I^{r-1} x^{-r+2} \subsetneq I^{r} x^{-r+1}=I^{r+1} x^{-r}=\ldots
$$

Hence $\widetilde{I}=I^{r} x^{-r+1}$. By Proposition 2.1 , we have $\widetilde{I}=\left(I^{r}: I^{r-1}\right)$, thus it is enough to prove that $\left(I^{r-1}: I^{r-2}\right) \subsetneq\left(I^{r}: I^{r-1}\right)$.

Since $x I^{r-1} \subsetneq I^{r}$, there exists an element $a \in I^{r}$ such that $a / x \notin I^{r-1}$ (and hence $\left.a / x^{r-1} \notin I\right)$.

Since $I \subseteq \mathfrak{C}$, then $a / x^{r-1} \in \widetilde{I} \subseteq R$; moreover, if $b \in I^{r-1}$, then $a b / x^{r-1} \in$ $I^{2 r-1} x^{-(r-1)}=I^{r} x^{r-1} x^{-(r-1)}=I^{r}$. Therefore $a / x^{r-1} \in\left(I^{r}: I^{r-1}\right)$. On the other hand $\left(a / x^{r-1}\right) x^{r-2}=a / x \notin I^{r-1}$, hence $a / x^{r-1} \notin\left(I^{r-1}: I^{r-2}\right)$.

Corollary 3.3: $\quad$ Suppose $R$ is a reduced Noetherian ring as in (3.1) and $I$ is a regular ideal of $R$ having a principal reduction. If $I \subseteq \mathfrak{C}$, then $I$ is stable if and only if $I=\widetilde{I}$. 
Proof: The assertion is clear if $I$ is a principal ideal. If $I$ is not principal, then by Theorem 3.2, the reduction number $r$ of $I$ is 1 if and only if the Ratliff-Rush number $k$ of $I$ is zero, i.e., if and only if $\widetilde{I}=(I: R)=I$.

Corollary 3.4: Suppose $R$ is a reduced Noetherian ring as in (3.1) and $I$ is a regular ideal of $R$ having a principal reduction. If $I \subseteq \mathfrak{C}$ is Ratliff-Rush closed and non-principal, then I has relation type 2.

Proof: Apply Corollary 3.3 and Corollary 2.10 .

Remark 3.5: We would like to take this opportunity to remark that (1.15) of [HJLS, page 363] is incorrect. It is asserted there that, if $R$ is a one-dimensional semilocal domain and $I$ is an ideal of $R$ contained in the conductor, then $I$ is stable. This assertion is false without the additional hypothesis that $I$ is a Ratliff-Rush ideal. A simple example that illustrates this is to let $t$ be an indeterminate over a field $K$ and let $R=K\left[t^{3}, t^{4}, t^{5}\right]_{\left(t^{3}, t^{4}, t^{5}\right)}$. The maximal ideal $\mathfrak{m}=\left(t^{3}, t^{4}, t^{5}\right) R$ coincides with the conductor. Note however that the ideal $I=\left(t^{3}, t^{4}\right) R$ is not Ratliff-Rush, so is not stable.

We also remark that for $R$ a Noetherian domain, Corollary 3.3 follows from [HJL, (4.7)] which asserts that if $D$ is a Noetherian integral domain and $I$ is an ideal of $D$ that is integral over a principal ideal generated by an element of the conductor, then the Ratliff-Rush ideal $\widetilde{I}$ associated to $I$ is stable.

Notice that, if $x R$ is a principal reduction of $I$, then

$$
\widetilde{I^{m}}=I^{m s} x^{-m(s-1)} \cap R
$$

for every $s \in \mathbb{N}$ such that $s \geq r$, where $r$ the reduction number of $I$. Using this observation we investigate conditions sufficient to imply the equality $\widetilde{I^{n+1}} \cap I^{n}=$ $I^{n} \widetilde{I}$ (cf. [HJLS Questions 1.16]). Naturally the inclusion $\widetilde{I^{n+1}} \cap I^{n} \supseteq I^{n} \widetilde{I}$ always holds, since $I^{n} \widetilde{I} \subseteq \widetilde{I^{n}} \widetilde{I}$ and $\left\{\widetilde{I^{n}}\right\}_{n \geq 0}$ is a filtration.

Theorem 3.6: $\quad$ Suppose $R$ is a reduced Noetherian ring as in (3.1) and $I$ is a regular ideal of $R$ having a principal reduction $x R$. If $I \subseteq \mathfrak{C}$, then $\widetilde{I^{n+1}}=I^{n} \widetilde{I}$, for every $n \geq 0$. In particular, since $I^{n} \widetilde{I} \subseteq I^{n}$, we have

$$
\widetilde{I^{n+1}} \cap I^{n}=I^{n} \widetilde{I}
$$


Proof: Since $I \subseteq \mathfrak{C}$, we have, for all sufficiently large $s \in \mathbb{N}$,

$$
\widetilde{I^{n+1}}=I^{(n+1) s} x^{-(n+1)(s-1)}=I^{n s+s} x^{-n s+n-s+1}
$$

and

$$
I^{n} \widetilde{I}=I^{n} I^{s} x^{-s+1}=I^{n+s} x^{-s+1} .
$$

Moreover, if $s+n \geq r$, we have

$$
I^{n+s} x^{-s+1}=I^{n+s} I^{n(s-1)} x^{-s+1} x^{-n(s-1)}=\widetilde{I^{n+1}} .
$$

Concerning the relationship of $\widetilde{I^{n+1}} \cap I^{n}$ with $I^{n} \widetilde{I}$ and $I^{n+1}$, we note the following:

Corollary 3.7: Suppose $R$ is a reduced Noetherian ring as in (3.1) and $I$ is a regular ideal of $R$ contained in $\mathfrak{C}$ with a principal reduction $x R$ and reduction number $r$. Then, for any integer $n \geq r-1, \widetilde{I^{n+1}} \cap I^{n}=I^{n} \widetilde{I}=I^{n+1}$.

Proof: This is immediate from Proposition 2.2 and the fact that $I^{n} \widetilde{I} \subseteq \widetilde{I^{n+1}}$.

Example shows $I^{n} \widetilde{I}$ may be properly contained in $\widetilde{I^{n+1}} \cap I^{n}$ for $n<r-1$.

Recall that a filtration $\left\{F_{n}\right\}_{n \geq 0}$ of a ring $R$ is called a good filtration if there exists an ideal $I$ of $R$ such that $I F_{i} \subseteq F_{i+1}$, for all $i \geq 0$, and if $I F_{n}=F_{n+1}$, for all $n$ large enough. (cf.[HZ Definition 2.1]). Hoa and Zarzuela in [HZ, Example 2.3] show that the Ratliff-Rush filtration is a good filtration. For a good filtration it is possible to define the notions of reduction, minimal reduction and reduction number (cf. [HZ, Definitions 2.5 and 3.1]). In the hypotheses of this section, if $I$ has a principal reduction $x R$, then $\left\{x^{n} R\right\}_{n \geq 0}$ is a minimal reduction for $\left\{\widetilde{I^{n}}\right\}_{n \geq 0}$ (cf.[HZ, Proposition 2.6]). The reduction number of the Ratliff-Rush filtration $\left\{\widetilde{I^{n}}\right\}_{n \geq 0}$ with respect to $\left\{x^{n} R\right\}_{n \geq 0}$ is defined to be the minimum integer $s$ such that $\widetilde{I^{n+1}}=x \widetilde{I^{n}}$ for all $n \geq s$. This integer $s$ is independent of the principal reduction $x R$. It may also be characterized as the smallest nonnegative integer $s$ such that $\widetilde{I^{s+1}} \subseteq x R$, for $\widetilde{I^{n+1}} \subseteq x R$ implies $\widetilde{I^{n+1}}=x \widetilde{I^{n}}$.

It is easy to see that if $r$ is the reduction number of $I$ and $s$ the reduction number of the filtration $\left\{\widetilde{I^{n}}\right\}_{n \geq 0}$, then $s \leq r$. For if $I^{n+1}=x I^{n}$, then $I^{n}$ and $I^{n+1}$ are both stable and hence Ratliff-Rush ideals as noted in Remark 2.4 , so $\widetilde{I^{n+1}}=x \widetilde{I^{n}}$. Example shows that the reduction number $r$ of $I$ may be strictly bigger than $s$. 
We prove that inclusion in the conductor implies a strict upper bound on the reduction number of a Ratliff-Rush filtration.

Theorem 3.8: $\quad$ Suppose $R$ is a reduced Noetherian ring as in (3.1) and $I$ is a regular ideal of $R$ having a principal reduction $x R$ and reduction number $r$. If there exists an integer $m$ such that $x^{m} \in \mathfrak{C}$, then, for any integer $n \geq m, \widetilde{I^{n+1}}=x \widetilde{I^{n}}$. In particular, if $s$ is the reduction number of the filtration $\left\{\widetilde{I^{n}}\right\}_{n \geq 0}$, then $s \leq m$.

Proof: If $m \geq r$, the assertion is clear, so we assume that $m<r$. As we mentioned immediately before Theorem 3.6

$$
x \widetilde{I^{n}}=x\left(I^{n s} x^{-n(s-1)} \cap R\right)
$$

and

$$
\widetilde{I^{n+1}}=I^{(n+1) s} x^{-(n+1)(s-1)} \cap R .
$$

Hence, to prove that $\widetilde{I^{n+1}} \subseteq x \widetilde{I^{n}}$ (the other inclusion is always true), we have to prove that any element of $R$ of the form $y / x^{n s-n+s-1}$, where $y \in I^{n s+s}$, belongs to $x R$. We may assume $n s+s>r$; hence we have $I^{n s+s}=x^{n s+s-r} I^{r}$. It follows that there exists $z \in I^{r}$ such that $y / x^{n s-n+s}=z / x^{r-n}=x^{n} z / x^{r}$. Since $n \geq m$, $x^{n} \in \mathfrak{C}$; hence $y / x^{n s-n+s} \in R$ and $y / x^{n s-n+s-1} \in x R$.

Corollary 3.9: $\quad$ Suppose $R$ is a reduced Noetherian ring as in (3.1) and $I$ is a regular ideal of $R$ having a principal reduction $x R$ and reduction number $r$. If $I \subseteq \mathfrak{C}$, then $\widetilde{I^{n+1}}=x \widetilde{I^{n}}$ for all $n \geq 1$, so the reduction number of the filtration $\left\{\widetilde{I^{n}}\right\}_{n \geq 0}$ is less than or equal to 1 .

Proposition 3.10: $\quad$ Suppose $R$ is a reduced Noetherian ring as in (3.1) and $I$ is a regular ideal of $R$ having a principal reduction $x R$ and reduction number $r$. If $I \subseteq \mathfrak{C}$ and $I$ is not Ratliff-Rush closed, then the asymptotic Ratliff-Rush number, $h$, coincides with $r$.

Proof: In this case, one has $\widetilde{I^{h-1}}=\left(I^{h}: x\right)$ where $h \geq 2$ is the asymptotic RatliffRush number of $I$. In fact, by the definition of $h$, we have $\widetilde{I^{h-1}} \neq I^{h-1}$ and $\widetilde{I^{n}}=I^{n}$ for all $n \geq h$. By Corollary $3.9 I^{h}=\widetilde{I^{h}}=x \widetilde{I^{h-1}}$ and we get $\widetilde{I^{h-1}} \subseteq\left(I^{h}: x\right)$ from which the conclusion. Since $I \subsetneq \widetilde{I}$, we have $r \geq 2$ and we deduce that $I^{r} \subseteq x R$. In fact $I^{r}=\widetilde{I^{r}}$ by Proposition 2.2 and $\widetilde{I^{r}}=x \widetilde{I^{r-1}}$ by Corollary 3.9. Also by 
definition of $r$ we have $x I^{r-1} \subsetneq I^{r}$. Hence $x I^{r-1} \subsetneq I^{r} \cap x R$ and $h=r$ follows by Proposition 2.7.

For $J=x R$ a principal reduction of $I$, we note the following case where the integer $k_{J}$, the Ratliff-Rush number of $I$ with respect to $J$, is equal to the RatliffRush number of $I$, and therefore independent of the minimal reduction chosen.

Corollary 3.11: $\quad$ Suppose $R$ is a reduced Noetherian ring as in (3.1) and $I$ is a regular ideal of $R$ having a principal reduction $x R=J$ and reduction number $r$. If $I \subseteq \mathfrak{C}$ and $I \subsetneq \widetilde{I}$, then $k_{J}=k=r-1$.

Proof: By Theorem 3.2, we have $k=r-1$. Since $I \subsetneq \widetilde{I}, r \geq 2$. If $r=2$, then $k=1$. Since $k_{J} \leq k$ and $k_{J} \neq 0$, we have $k_{J}=k$ in this case.

Suppose $r \geq 3$. Since $h=r$, we have $x^{h-2} \widetilde{I}=\widetilde{I^{h-1}}=\left(I^{h}: x\right)$. To show $k_{J}=k$, it suffices to show that $\left(I^{r-1}: x^{r-2}\right)$ is strictly contained in $\left(I^{r}: x^{r-1}\right)$. This is true since otherwise we would have $\left(I^{r-1}: x^{r-2}\right)=\widetilde{I}$ and we would obtain $\widetilde{I^{r-1}}=x^{r-2} \widetilde{I} \subseteq I^{r-1}$, which is impossible since $h=r$.

\section{Applications and examples in the one-dimensional case.}

Notation 4.1: $\quad$ Suppose $(R, \mathfrak{m})$ is a one-dimensional Cohen-Macaulay local ring such that the residue field $R / \mathfrak{m}$ of $R$ is infinite and the integral closure $\bar{R}$ of $R$ in its total ring of fractions $Q(R)$ is a finitely generated $R$-module. The conductor $\mathfrak{C}=\left(R:_{Q(R} \bar{R}\right)$ of $\bar{R}$ into $R$ is a regular ideal.

Note that if $(R, \mathfrak{m})$ is a one-dimensional Cohen-Macaulay local ring having the property that its integral closure $\bar{R}$ is a finitely generated $R$-module, then $R$ is reduced. For if $a \in R$ is a nilpotent element, let $b \in \mathfrak{m}$ be a regular element. Then $a / b^{n} \in Q(R)$ is integral over $R$ for each positive integer $n$. Hence $a \in \cap_{n=1}^{\infty} b^{n} \bar{R}=$ (0), the last equality because $b$ is in the Jacobson radical of the Noetherian $\operatorname{ring} \bar{R}$.

Proposition 4.2: $\quad$ With notation as in (4.1), if $I \subseteq \mathfrak{C}$ is an $\mathfrak{m}$-primary ideal and $\tilde{I} \supsetneq I$, then $I$ has Ratliff-Rush number $k \leq \lambda=l_{R}(\widetilde{I} / I)$.

Proof: Since $I \subseteq \mathfrak{C}$, by Theorem 3.2, $I$ has reduction number $r=k+1$. Moreover, as noted at the beginning of the previous section, $\widetilde{I}=I^{r} x^{-r+1}$ and we have the 
following chain of ideals:

$$
I \subsetneq I^{2} x^{-1} \subsetneq \cdots \subsetneq I^{r-1} x^{-r+2} \subsetneq I^{r} x^{-r+1}=\widetilde{I} .
$$

In this chain there are $r-1=k$ steps; hence $\lambda=l_{R}(\widetilde{I} / I) \geq k$.

With $(R, \mathfrak{m})$ as in (4.1), if $I$ is an $\mathfrak{m}$-primary ideal of $R$, then the associated graded ring $G(I)=R[I t] / I R[I t]$ is a one-dimensional graded ring having a unique maximal graded ideal. The 0 -th local cohomology of $G(I)$ with respect to this maximal ideal (or equivalently with respect to the ideal $G(I)_{+}$consisting of elements of positive degree) is the ideal of $G(I)$ of elements that annihilate a power of this maximal ideal (or equivalently a power of $G(I)_{+}$). This is a graded ideal and is zero if and only if $G(I)$ is Cohen-Macaulay (since we are in dimension one). Proposition 3.6 provides information on this 0 -th local cohomology.

Proposition 4.3: $\quad$ Let $R$ and $I \subseteq \mathfrak{C}$ be as in (4.1). Then

$$
H_{G(I)_{+}}^{0}(G(I))=\widetilde{I} / I \oplus I \widetilde{I} / I^{2} \oplus \cdots \oplus I^{r-2} \widetilde{I} / I^{r-1}
$$

where $r$ is the reduction number of $I$.

Proof: Recalling that

$$
H_{G(I)_{+}}^{0}(G(I))=\bigoplus_{n \geq 0} \frac{\widetilde{I^{n+1}} \cap I^{n}}{I^{n+1}},
$$

the result follows by Proposition 3.6 and Proposition 3.2.

The following corollary is now immediate from Proposition 4.2 and Proposition 4.3.

Corollary 4.4: Let $R$ and $I \subseteq \mathfrak{C}$ be as in (4.1). If $\lambda=l_{R}(\widetilde{I} / I)=1$, then $I$ has Ratliff-Rush number $k=1, \widetilde{I}=\left(I^{2}: I\right)$, the reduction number of $I$ is $r=2$ and $H_{G(I)_{+}}^{0}(G(I))=\widetilde{I} / I$.

In what follows we provide a set of examples regarding the results given in the previous sections. The example are all complete one-dimensional local Noetherian domains of the form $R=K \llbracket t^{s}: s \in S \rrbracket$, i.e. formal power series in the indeterminate $t$ with coefficients in a field $K$ and exponents from an additive submonoid $S$ of the nonnegative integers that contains all sufficiently large integers. The formal power 
series ring $K \llbracket t \rrbracket$ is a finitely generated $R$-module having the same fraction field as $R$. Thus, in each of the examples, the notation in (4.1) is valid if we assume the field $K$ is infinite. Since the ideals we consider are generated by monomials $t^{s}$, the examples are valid even if the field $K$ is finite.

Example 4.5: An ideal $I$ such that $I=\left(I^{2}: I\right)$, but $\widetilde{I} \supsetneq\left(I^{2}: I\right)$.

Set $R=K \llbracket t^{4}, t^{5}, t^{6}, t^{7} \rrbracket$ and $I=\left(t^{4}, t^{5}\right)$. Observe that $I^{2}=\left(t^{8}, t^{9}, t^{10}\right)$ and $I^{3}=\left(t^{12}, t^{13}, t^{14}, t^{15}\right)$. Thus $\widetilde{I}=\mathfrak{C}=\mathfrak{m}$ and $\widetilde{I}=\left(I^{3}: I^{2}\right)$ while $\left(I^{2}: I\right)=I$.

Example 4.6: An ideal $I \nsubseteq \mathfrak{C}$ such that $k<r-1$. (cf. Proposition 2.1and Theorem 3.2).

Let $R=K \llbracket t^{5}, t^{7}, t^{23} \rrbracket$ and $I=\left(t^{5}, t^{7}\right)$. We have $I^{5}=t^{5} I^{4}$, hence $r=4$, while $k=1$, since $\widetilde{I}=\mathfrak{m}=\left(I, t^{23}\right)=\left(I^{2}: I\right)$. Notice that, in this case, $\lambda=1$.

Example 4.7: $\quad$ An ideal $I$ such that $h(I)<r(I)$. (cf. Proposition 2.2and Proposition 2.7).

Let $R=K \llbracket t^{3}, t^{5} \rrbracket$ and $I=\mathfrak{m}$. In this case each power of $I$ is a Ratliff-Rush ideal, so $h(I)=0$. However, $\mathfrak{m}^{2} \supsetneq t^{3} \mathfrak{m}$, since $t^{10} t^{-3} \notin \mathfrak{m}$. Hence $r(I) \geq 2$.

Example 4.8: $\quad$ An ideal $I \subset \mathfrak{C}$ such that $N(I)=3$ and $r(I)=4$.

Let $R=K \llbracket t^{9}, t^{10}, t^{11}, t^{12}, t^{13}, t^{14}, t^{15}, t^{16}, t^{17} \rrbracket$ and $I=\left(t^{9}, t^{10}, t^{12}\right)$. Using the computer algebra program MACAULAY one sees that $N(I)=3$ and $r=4$. These facts can also be checked by hand.

Example 4.9: A nonstable ideal $I \nsubseteq \mathbb{C}$ such that $\widetilde{I}=I$. (cf. Remark 2.4 and Corollary 3.3).

Set $R=K \llbracket t^{3}, t^{4} \rrbracket$ and $I=\mathfrak{m} . \quad I$ is not stable, since $t^{8} \in I^{2} \backslash t^{3} I$. In this case $r=2$ and $k=0$.

Example 4.10: An example that shows $I^{n} \widetilde{I}$ may be properly contained in $\widetilde{I^{n+1}} \cap$ $I^{n}$. (cf. Theorem 3.6).

Let $R=K \llbracket t^{6}, t^{7}, t^{15}, t^{16}, t^{17} \rrbracket$ and $I=\widetilde{I}=\mathfrak{m}$. Then we have $I^{2}=\left(t^{12}, t^{13}, t^{14}, t^{22}, t^{23}\right)$, $I^{3}=\left(t^{18}, t^{19}, t^{20}, t^{21}, t^{29}\right), I^{4}=\left(t^{24}, t^{25}, t^{26}, t^{27}, t^{28}\right), I^{5}=t^{30} \bar{R}$ and $I^{5+m}=t^{6 m} I^{5}$ for any $m \geq 0$. Clearly this implies that $r(I)=5$. Moreover, for each $m \geq 2$, $\widetilde{I^{m}}=t^{6 m} \bar{R}$ and $\widetilde{I^{m}} \cap I^{m-1}=\widetilde{I^{m}}$. 
Hence $I I \subsetneq \widetilde{I^{2}}, I^{2} I \subsetneq \widetilde{I^{3}}, I^{3} I \subsetneq \widetilde{I^{4}}$ and $I^{4} I=\widetilde{I^{5}}$. Notice also that $I^{2} \subseteq \mathfrak{C}=$ $t^{12} \bar{R}$.

Example 4.11: $\quad$ An ideal $I$ with reduction number greater than the reduction number of the Ratliff-Rush filtration. (cf. Proposition 3.8).

Let $R=K \llbracket t^{4}, t^{5}, t^{11} \rrbracket$ and $I=\mathfrak{m}$. We have $\mathfrak{m}^{4}=t^{4} \mathfrak{m}^{3}$ and $t^{15} \in \mathfrak{m}^{3} \backslash t^{4} \mathfrak{m}^{2}$ Hence $r=3$. Moreover $\mathfrak{m}$ is Ratliff-Rush closed and $\widetilde{\mathfrak{m}^{2}}=\left(t^{8}, t^{9}, t^{10}, t^{11}\right)$; hence $\widetilde{\mathfrak{m}^{n+1}}=t^{4} \widetilde{\mathfrak{m}^{n}}$ for all $n \geq 2$. Notice that $\left(t^{4}\right)^{2} \in \mathfrak{C}=t^{8} K \llbracket t \rrbracket$.

Example 4.12: $\quad$ An ideal $I \nsubseteq \mathfrak{C}$ with a principal reduction $J=(x)$ such that $k_{J}<k$. (cf. Corollary 3.11).

Let $R=K \llbracket t^{4}, t^{5}, t^{6} \rrbracket$ and $I=\left(t^{4}, t^{5}\right)$. We have: $I^{2}=t^{8} K+t^{9} K+t^{10} K+$ $t^{12} K \llbracket t \rrbracket \subsetneq \widetilde{I^{2}}=t^{8} K \llbracket t \rrbracket$ and $I^{3}=t^{12} k \llbracket t \rrbracket=\widetilde{I^{3}}$. This implies that $r=h=3$; moreover $\left(I^{2}: I\right)=I,\left(I^{3}: I^{2}\right)=\mathfrak{m}=\widetilde{I}$ and $\left(I^{2}: t^{4} R\right)=\mathfrak{m}$. Hence if $J=t^{4} R$, $k_{J}=1$, while $k=2$.

Notice that $t^{15} \in\left(I \cap t^{4} R\right.$ ) does not belong to $t^{4} I^{2}$ (cf. Proposition 2.7).

Example 4.13: An ideal $I \nsubseteq \mathfrak{C}$ such that $k=3>\lambda=1$. (cf. Proposition 4.2).

Set $R=K \llbracket t^{5}, t^{6}, t^{8} \rrbracket$ and $I=\left(t^{5}, t^{6}\right)$. We have $\widetilde{I}=\mathfrak{m}=\left(I^{4}: I^{3}\right)$; hence $\lambda=1$, while $k=3$ (notice that, in this case, $I^{5}=t^{5} I^{4}$, hence $r=4=k+1$ ).

This is also an example of an ideal such that $I^{2} x^{-1} \supsetneq I$, but $I^{2} x^{-1} \cap R=I$ (cf. proof of Proposition 4.2). In fact we have $I^{2}=\left(t^{10}, t^{11}, t^{12}\right)$, hence $t^{7} \in I^{2} t^{-5}$ does not belong to $I^{2} t^{-5} \cap R$.

Example 4.14: An ideal $I \subset \mathfrak{C}$ such that $k<\lambda$. (cf. Proposition 4.2).

Set $R=K \llbracket t^{5}, t^{6}, t^{7}, t^{8}, t^{9} \rrbracket$ and $I=\left(t^{5}, t^{6}, t^{7}\right)$. Since $I^{2}=\left(t^{10}, t^{11}, t^{12}, t^{13}, t^{14}\right)$ and $\widetilde{I}=\mathfrak{m}$, then $\widetilde{I}=\left(I^{2}: I\right), k=1$ and $\lambda=2$.

\section{REFERENCES}

[ES] P. Eakin and A. Sathaye, Prestable ideals, J. Algebra 41 (1976), 439-454.

[HJLS] W. Heinzer, B. Johnston, D Lantz and K. Shah, Coefficient ideals in and blowups of a commutative Noetherian domain, J. Algebra 162 (1993), 355-.

[HJL] W. Heinzer, B. Johnston and D Lantz, First coefficient domains and ideals of reduction number one, Comm. Alg. 21(10) (1993), 3797-3827.

[HLS] W. Heinzer, D Lantz and K. Shah, The Ratliff-Rush ideals in a Noetherian ring, Comm. Alg. 20(2) (1992), 591-622.

[HZ] L.T. Hoa and S. Zarzuela, Reduction number and a-invariant of good filtrations, Comm. Alg. 22(14) (1994), 5635-5656.

[Hu1] S. Huckaba, Reduction numbers for ideals of analytic spread one, J. Algebra 108 (1987), 503-512. 
[Hu2] S. Huckaba, Reduction numbers for ideals of higher analytic spread, Proc. Camb. Phil. Soc. 102 (1987), no. 49, 49-57.

[H] C. Huneke, Hilbert functions and symbolic powers, Michigan Math. J. 34 (1987), 293318.

[I] S. Itoh, Integral closures of ideals generated by regular sequences, J. Algebra 117 (1988), 390-401.

[L] J. Lipman, Stable ideals and Arf rings, Amer. J. Math. 93 (1971), 649-685.

[NR] D. G. Northcott and D. Rees, Reductions of ideals in local rings, Proc. Camb. Phil. Soc. 50 (1954), 145-158.

[RR1] L. J. Ratliff, Jr. and David E. Rush, Two notes on reductions of ideals, Indiana Univ. Math. J. 27 (1978), 929-934.

[RR2] L. J. Ratliff, Jr. and David E. Rush, A bracket power characterization of analytic spread one ideals, Trans. Amer. Math. Soc., to appear.

[RV] M.E. Rossi and G. Valla, Cohen-Macaulay local rings of embedding dimension $e+d-3$, Proc. London Math. Soc., to appear.

[SV] J. Sally and W. Vasconcelos, Stable rings, J. Pure Appl. Algebra 4 (1974), 319-336.

[VV] P. Valabrega and G. Valla, Form rings and regular sequences, Nagoya Math. J. 72 (1978), 93-101.

[V] W. Vasconcelos, The reduction number of an algebra, Composito Math. 104 (1996), 189-197.

[W] Y. Wu, Hilbert functions, reduction numbers and relation types, Ph.D. Thesis, Purdue Univ. (1992).

Università di Catania - Dipartimento di Matematica,

Viale Andrea Doria, 6 - 95125 Catania, Italy

E-mail: mdanna@dipmat.unict.it

\section{Università di L'Aquila - Dipartimento di Matematica, \\ Via Vetoio, Loc. Coppito - 67100 L'Aquila, Italy}

E-mail: guerran@univaq.it

Purdue University - Department of Mathematics,

West Lafayette, Indiana 47907, USA

\section{E-mail: heinzer@math.purdue.edu}

${ }^{1} 1991$ Mathematics Subject Classification. 13A30, 13C05, 13E05, 13H15

${ }^{2}$ Key words and phrases. Ratliff-Rush filtration, principal reductions, conductor. 